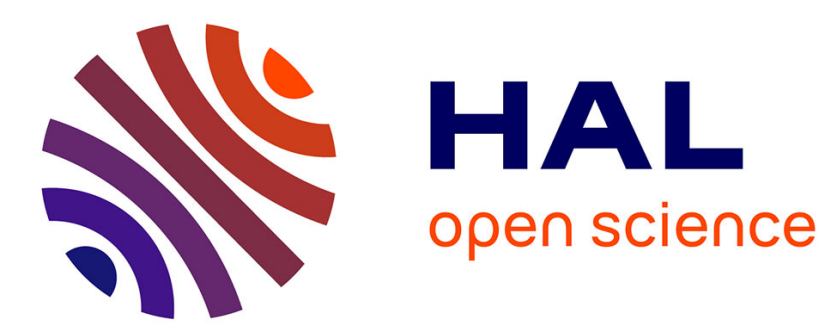

\title{
Changing the routing protocol without transient loops
} Nancy El Rachkidy, Alexandre Guitton

\section{To cite this version:}

Nancy El Rachkidy, Alexandre Guitton. Changing the routing protocol without transient loops. Computer Communications, 2016, 82, pp.49 - 58. 10.1016/j.comcom.2016.02.010 . hal-01448127

\section{HAL Id: hal-01448127 https://hal.uca.fr/hal-01448127}

Submitted on 27 Jan 2017

HAL is a multi-disciplinary open access archive for the deposit and dissemination of scientific research documents, whether they are published or not. The documents may come from teaching and research institutions in France or abroad, or from public or private research centers.
L'archive ouverte pluridisciplinaire HAL, est destinée au dépôt et à la diffusion de documents scientifiques de niveau recherche, publiés ou non, émanant des établissements d'enseignement et de recherche français ou étrangers, des laboratoires publics ou privés. 


\title{
Changing the Routing Protocol without Transient Loops
}

\author{
Nancy El Rachkidy, Alexandre GuitTon* \\ Clermont Université, Université Blaise Pascal, LIMOS, BP 10448, F-63000 Clermont-Ferrand, France \\ CNRS, UMR 6158, LIMOS, F-63173 Aubière, France
}

\begin{abstract}
Computer networks generally operate using a single routing protocol. However, there are situations where the routing protocol has to be changed (e.g., because an update of the routing protocol is available, or because an external event has triggered a traffic with different quality of service requirements). In this paper, we show that an uncontrolled change of the routing protocol might yield to transient routing loops (even if the involved routing protocols are loop-free). We show that it is possible to achieve a loop-free change for multiple destinations using a strongly connected component approach producing successive steps, where each step contains nodes that can change the routing protocol in parallel. Our aim is to reduce the number of steps in order to reduce the time required for the network to change from one routing protocol to another. Simulation results show that our strongly connected component approach greatly reduces the number of steps compared to the state of the art, and thus it greatly reduces the time for the change.
\end{abstract}

Keywords: Routing protocols, routing protocol change, transient routing loops.

\section{Introduction}

Computer networks generally operate a single routing protocol which determines the route packets have to follow in order to reach a destination. However, some situations require to change the current routing protocol. For example, this change might be triggered by the availability of a major update of the protocol or the correction of a security issue [1]. Another example concerns monitoring applications in wireless sensor networks, where the detection of a critical event might trigger the change from an energy-efficient routing protocol to a delay-sensitive routing protocol [2]. Another example focuses on the changes in routing decisions caused by major modifications of the topology (due to link or node failures, or to significant changes in routing metrics) $[3,4,5]$.

If nodes are accurately synchronized, they can perform the change simultaneously from the current routing protocol $\mathcal{R}_{1}$ to the new routing protocol $\mathcal{R}_{2}$. However, this solution is often difficult to implement in practice, especially in large networks. Indeed, the cost of an accurate synchronization might be prohibitive, or nodes might be operated by different network administrators, leading to different plannings for the change. We assume in the following that nodes cannot be synchronized in such a precise manner.

If nodes are not synchronized and if nodes perform the change arbitrarily, transient routing loops might occur, even if the routing protocols are loop-free when considered independently. Figure 1 shows such an example. Initially, all packets towards $d$ are routed according to routing protocol $\mathcal{R}_{1}$, and $\mathcal{R}_{1}$ is loop-free (see Figure 1(a)). If nodes are requested to change to another protocol $\mathcal{R}_{2}$ in arbitrary order $\left(\mathcal{R}_{2}\right.$ is shown on Figure 1(b)), it is possible that $c$ changes first, resulting into the routing depicted on Figure 1(c). In this case, a transient routing loop occurs between nodes $b$ and $c$. This loop will eventually disappear when node $b$ changes to $\mathcal{R}_{2}$ too, but the impact on the network performance is not negligible.

\footnotetext{
* Corresponding author.

Email Address: alexandre.guitton@univ-bpclermont.fr (Alexandre GUITTON)

Phone: +33 473405229, Fax: +33 473407639
} 


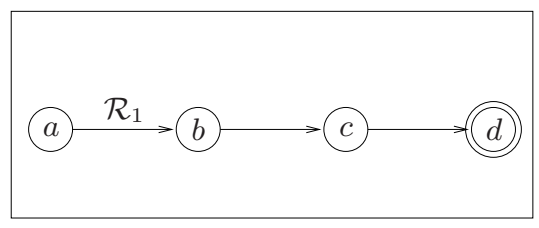

(a)

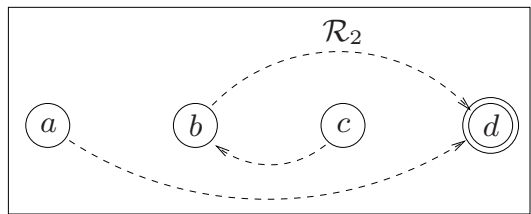

(b)

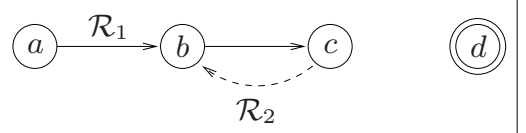

(c)

Figure 1: Uncontrolled changes might yield to routing loops. (a) The initial routing protocol $\mathcal{R}_{1}$ is loop-free for destination $d$. (b) The new routing protocol $\mathcal{R}_{2}$ is also loop-free for destination $d$. (c) A loop occurs between nodes $b$ and $c$, if $a$ and $b$ route according to $\mathcal{R}_{1}$ and $c$ routes according to $\mathcal{R}_{2}$.

Routing loops reduce network performance as they can cause node inaccessibility issues or overload the network. Even if the routing loops caused by the changes are transient (because all nodes will eventually perform the change to the new, loop-free routing protocol), our aim is to completely avoid them, as they might have a significant impact for the applications.

In [6], we classified routing protocols into three categories: (i) compatible routing protocols, which do not yield to routing loops when they are used together, (ii) delayable routing protocols, where nodes might avoid loops based on the knowledge of the distance functions of the two protocols, and (iii) combined routing protocols, when the distance functions of the two protocols are not known or hard to compute locally by the nodes. In [7], we use a probabilistic approach to avoid loops. Indeed, some nodes choose randomly whether to forward packets or to hold them. However, the common assumption of $[6,7]$ is that routing protocols alternate. In this paper, we make a more general assumption, where the change is final: once a node has changed to $\mathcal{R}_{2}$, it does not change back to $\mathcal{R}_{1}$ anymore. This new assumption makes the previous solutions inapplicable. Moreover, we show that the change to $\mathcal{R}_{2}$ can be performed in successive steps, where all nodes of the same step can perform the change arbitrarily without causing loops.

Our contribution is three-fold. First, we show that the probability of transient loops is high for both random and real topologies, and for several types of routing protocols pairs. Second, we improve the main heuristic proposed by [8] for networks with several destinations, by proposing a greedy mechanism to deal with troublesome destinations, and by computing a sequence of steps rather than a sequence of nodes. Third, we propose our centralized heuristic based on the computation of strongly connected components in order to classify nodes into steps. It is aimed at reducing the overall number of steps and thus reducing the change duration, which is our main metric.

The remainder of the paper is organized as follows. Section 2 describes relevant research works of the literature. Section 3 first presents two improvements for the main protocol of the literature [8]: a greedy mechanism for the per-destination ordering, and the computation of a sequence of steps rather than a sequence of nodes. Then, it presents our central theorem based on strongly connected components. Finally, it presents our heuristic based on this theorem. Section 4 evaluates the performance of these heuristics on several topologies and for several routing protocols pairs. Finally, Section 5 concludes this work.

\section{Related work}

In this section, we first describe architectures where routing loops might occur. Then, we present the solutions from the literature that avoid routing loops occurring during the change from one protocol to another. Then, we describe in details the Routing Tree Heuristic (RTH) presented in [8], as it is the main heuristic to change routing protocols. Finally, we describe the main differences between RTH [8] and this paper.

\subsection{Networks and protocols with risks of loop occurrence}

Several routing protocols that combine different routing decisions have been proposed in the literature. In [9], the authors propose to combine a reactive routing protocol with a greedy geographical routing protocol. When a packet has to be forwarded, the reactive protocol establishes the whole route to the destination. The 
geographical protocol is used when the next-hop according to the reactive protocol becomes unreachable. Routing loops can occur if the geographical protocol forwards packets to a node that uses the reactive protocol. In [10], a routing protocol $R_{d}$ that reduces delay is combined with a routing protocol $R_{e}$ that reduces energy. $R_{d}$ and $R_{e}$ are used depending on the traffic produced by the application: urgent packets are forwarded according to $R_{d}$, while periodic packets are forwarded according to $R_{e}$. Routing loops can occur if an urgent packet reaches a node that has a limited energy and uses $R_{e}$. In [11], packets are given a priority based on traffic type. The next-hop of a packet is computed according to several parameters, including packet priority, number of hops to the destination, link quality for the next-hop, residual energy for the next-hop, load of the next-hop, etc. Routing loops can occur if the parameters used by a node are different from the parameters used by another node on the path to the destination.

Multi-purpose Wireless Sensor Networks (WSNs) [12] have been proposed to enable a single WSN deployment to support several applications. The main advantage of multi-purpose WSNs is that the cost of deployment is shared by all the applications. Several researchers have proposed protocols for multi-purpose WSNs $[13,14,15,16]$. In such networks, several routing protocols can be used simultaneously, because the large amount of applications yield to different requirements that cannot be met by a single routing protocol. However, dealing with several routing protocols might cause routing loops when the choice of the routing protocol is made locally by each node $[6,7]$.

\subsection{Heuristics that avoid routing loops}

The problem of avoiding transient loops during a change of routing protocols is a recent issue. We summarize here the main related works.

In $[6,7]$, nodes are synchronized and forward packets according to a schedule composed of two periods $p_{1}$ and $p_{2}$. During $p_{1}$, nodes forward packets according to $\mathcal{R}_{1}$, and during $p_{2}$, nodes forward packets according to $\mathcal{R}_{2}$. Routing loops can occur if nodes become desynchronized or if the decision to forward a packet according to $\mathcal{R}_{1}$ or $\mathcal{R}_{2}$ is made locally by each node, independently of the period. In [6], properties of pairs of routing protocols are studied, and three categories are identified: (i) compatible routing protocols, which do not yield to routing loops, (ii) delayable routing protocols, where nodes might avoid loops based on the knowledge of the distance functions of the two protocols, and (iii) combined routing protocols, where the distance functions of the two protocols are not known or hard to compute locally by the nodes. The last two categories require $\mathcal{R}_{1}$ and $\mathcal{R}_{2}$ to alternate, as some nodes hold packets indefinitely for one routing protocol. They are not suitable for a final change of routing protocol, as we consider in this paper. In [7], two heuristics are proposed to avoid loops or reduce their occurrences. In the first heuristic, all loops are avoided by forbidding some nodes to forward packets according to one routing protocol $\mathcal{R}_{i}$. In the second heuristic, a probabilistic approach is used to reduce the risk of loops: nodes that could potentially be involved in loops choose randomly whether to forward or to hold packets for one routing protocol. These two heuristics cannot be applied here because the change from $\mathcal{R}_{1}$ to $\mathcal{R}_{2}$ is final: once a node has performed the change to $\mathcal{R}_{2}$, it does not change back to $\mathcal{R}_{1}$.

In [4], the authors show that most routing protocols can produce transient routing loops after a topological change. They show that such loops can be avoided by having routers process routing updates in a specific order. Their mechanism is able to deal with link failures, new links, or updates on link metrics. The differences with this paper are the following: (i) we consider arbitrary protocols for $\mathcal{R}_{1}$ and $\mathcal{R}_{2}$, while [4] considers a single routing protocol on two similar topologies, (ii) we reduce the number of steps required for the change, while [4] provides an ordering of updates that does not cause loops, and (iii) in our proposition, each node is updated exactly once for each destination, while the algorithm of [4] might update the same node several times.

In $[3,5]$, the authors show that ordering the routing updates yields to additional message overhead and increases the change delay. They avoid transient routing loops by exploiting the existence of one forwarding table per interface. Messages arriving through unexpected interfaces are discarded, because they indicate a discrepancy between the view of the router and of its neighbors. Once all routers have the same view of the topology, the protocol converges and produces loop-free routes. The main difference with this paper is that we do not drop packets during the change to reduce the impact of loops, but we avoid changing the routing protocol of nodes if it causes a loop. 
In $[17,18]$, the authors show that transient routing loops that occur after topological changes can be avoided by applying a sequence of topology updates. Between two topology updates, the same routing protocol is used, but some link values are modified, which result into changes in routing decisions. They propose a protocol that minimizes the number of topology updates. The difference with this paper are the following: (i) we consider two different routing protocols on a single topology, while $[17,18]$ consider a single routing protocol applied on a sequence of updated topologies, (ii) we consider arbitrary routing protocols, while [17] considers changes on only one link and [18] considers changes concerning the links of a single router, and (iii) we reduce the number of steps to perform the change, where each step is a set of nodes and each node appears exactly once per destination, while $[17,18]$ attempts to minimize the number of topology updates to guarantee the convergence of a single routing protocol without transient loops.

In [8], the authors describe a heuristic which is based on similar assumptions as in this paper. We describe this heuristic in details in the next subsection.

\subsection{Routing Tree Heuristic [8]}

In [8], authors proposed ordering algorithms in order to avoid loops during the change from one routing protocol $\mathcal{R}_{1}$ to another routing protocol $\mathcal{R}_{2}$. They proposed a heuristic called Routing Tree Heuristic (RTH).

RTH consists of the following. For each destination $d$, the ordering constraints are computed separately. First, a greedy algorithm is used to compute the set $S_{d}$ of nodes that do not yield to loops in the network. A node is added to $S_{d}$ if and only if its next-hops using $\mathcal{R}_{1}$ and using $\mathcal{R}_{2}$ are already in $S_{d}$. Second, the set $\bar{V}_{d}$ of nodes is built by adding each node that does not have the same next-hops using $\mathcal{R}_{1}$ and $\mathcal{R}_{2}$. Third, RTH builds a set of constraints $C$ in the following way: for each path on $\mathcal{R}_{2}$ from a source node to the destination $d$, a constraint is generated for the last pair of nodes $(u, v)$ on this path such that $u \in \bar{V}_{d}$ and $u \notin S_{d}$. In this way, the change from $\mathcal{R}_{1}$ to $\mathcal{R}_{2}$ starts from the destination backwards (according to $\mathcal{R}_{2}$ ) to the sources, which guarantees that no loop occur on the path. This means that a node $n$ does not change before all its successors on $\mathcal{R}_{2}$ have already changed. Finally, RTH creates an acyclic directed graph $G_{C}$ from the set of constraints $C$, and the ordering is computed as a topological sort of $G_{C}$.

\subsection{Differences between RTH and our proposition}

The differences with this paper are the following:

- RTH changes the routing protocol of nodes one by one, so the duration of the change is proportional to the number of nodes in the network. However, we show here that it is possible to change the routing protocol of several nodes in parallel, without any loop occurrence. We use this parallelism to reduce the time required for the routing protocol change.

- RTH changes the routing protocol of nodes by following the arcs of $\mathcal{R}_{2}$ from the destination backwards to the sources. However, we show that it is possible to change the routing protocol for nodes independently of their position on the paths from sources to destinations according to $\mathcal{R}_{2}$. We use a strongly connected component approach to identify those nodes.

- The authors of RTH assume that there are few troublesome destinations, so RTH tries to compute the ordering for all destinations together when possible. However, we show that troublesome destinations are relatively frequent, so we decided to consider destinations independently.

\section{Fast changes of routing protocols}

The main criteria when performing changes of routing protocols without transient loops is the overall time required for the change. We consider in this paper that it is possible to perform the change as a sequence of steps, where nodes of each steps can change their routing protocol in parallel. Thus, the time required for the change is proportional to the number of steps, rather than to the number of nodes in the network.

In this section, we first describe improvements to RTH [8]: the first improvement is a greedy mechanism that deals with troublesome destinations, and the second improvement enables the support of steps. Second, we give a mathematical background for parallel steps based on strongly connected components. Third, we describe our heuristic based on this background. 


\subsection{Improvements of $R T H$}

We present here two improvements of RTH. The first improvement describes the per-destination ordering, when a per-router ordering does not exist due to troublesome destinations. The second improvement describes how to enable parallel changes in RTH.

\subsubsection{Improvement 1: Greedy mechanism of per-destination ordering in RTH}

The first improvement is a greedy mechanism of per-destination ordering. Note that this ordering can be applied when a per-router ordering exists (in this case, all destinations can be regrouped, and the result is the same as with the original RTH) as well as when a per-router ordering does not exist (in this case, several destinations are regrouped in a greedy manner, and troublesome destinations are processed independently). Authors of [8] mainly described the per-router ordering (which assumes that there is no troublesome destination), and explained that troublesome destinations can be processed independently from the other destinations. However, they did not propose an algorithm that identifies troublesome destinations, or that aggregates non-troublesome destinations (this was left as future work due to the fact that troublesome destinations were limited in their experimental evaluation, cf Subsection V.C of [8]).

The RTH heuristic (see Figure 8 of [8]) is modified in the following way. Initially, the set of troublesome destinations is initialized to $\emptyset$. In the main loop that considers each destination $d$, the constraint graph $G_{C}$ is computed using $V$ and the set of constraints $C$. If the new graph $G_{C}$, including the constraints generated by all previous non-troublesome destinations and the new destination $d$, has no loop, then the new destination is considered as a non-troublesome destination. However, if the new graph $G_{C}$ has a loop, destination $d$ is added to the list of troublesome destinations, and the constraints generated from this destination are removed from $C$. In this case, modifications to $G_{C}$ concerning $d$ are discarded. After all destinations have been considered, the ordering is made first for all non-troublesome destinations together using $G_{C}$, and then for all troublesome destinations one by one, using the per-destination ordering.

\subsubsection{Improvement 2: Parallel changes in RTH}

The second improvement consists of enabling parallel changes in RTH, in order to obtain a sequence of steps rather than a sequence of nodes. This modification can be performed efficiently from the constraint graph $G_{C}$ computed for all non-troublesome destinations (as well as for each troublesome destination independently). This improvement consists of changing the last step of RTH, by replacing the topological sort of $G_{C}$ with Algorithm 1. In this algorithm, a new set $S$ is added to sequence $T$ at each iteration. At each iteration, all nodes that have no incoming edges in $G_{C}$ (that is, no constraints), are added to the new set $S$ and all edges leaving from these nodes are removed from $G_{C}$. Since $G_{C}$ has no loop (due to the construction of all non-troublesome destinations, and to the fact that troublesome destinations are considered independently), the process ends with all nodes being in $T$. In our implementation, sequence $T$ contains first the steps for all non-troublesome destinations, then the steps for each troublesome destination successively.

In the following, we denote by RTH-p this heuristic.

\subsubsection{Example of RTH-p}

In the following, we describe RTH-p in an example. This example shows our greedy mechanism for per-destination ordering, as well as parallel changes.

Figure 2 shows a graph of five nodes, with three destinations and two routing protocols: (a) destination $a$ with $\mathcal{R}_{1}^{a}$ and $\mathcal{R}_{2}^{a}$, (b) destination $d$ with $\mathcal{R}_{1}^{d}$ and $\mathcal{R}_{2}^{d}$, and (c) destination $e$ with $\mathcal{R}_{1}^{e}$ and $\mathcal{R}_{2}^{e}$. When considering destinations independently, RTH produces the order $(a, e, d, c, b)$ for destination $a,(d, e, a, b, c)$ for destination $d$, and $(e, d, c, b, a)$ for destination $e$. When considering all destinations together, our greedy mechanism first considers destination $a$, identifies destination $d$ as troublesome, and identifies destination $e$ as non-troublesome. Thus, RTH-p produces sequence $(\{d, e\},\{c\},\{b\},\{a\})$ for both non-troublesome destinations $a$ and $e$, and sequence $(\{a, d, e\},\{b\},\{c\})$ for troublesome destination $d$. The resulting sequence is $(\{d, e\},\{c\},\{b\},\{a\},\{a, d, e\},\{b\},\{c\})$ (to simplify the notation, we did not indicate which destination is concerned by the change at each step). Note that each node appears several times but for different destinations. 


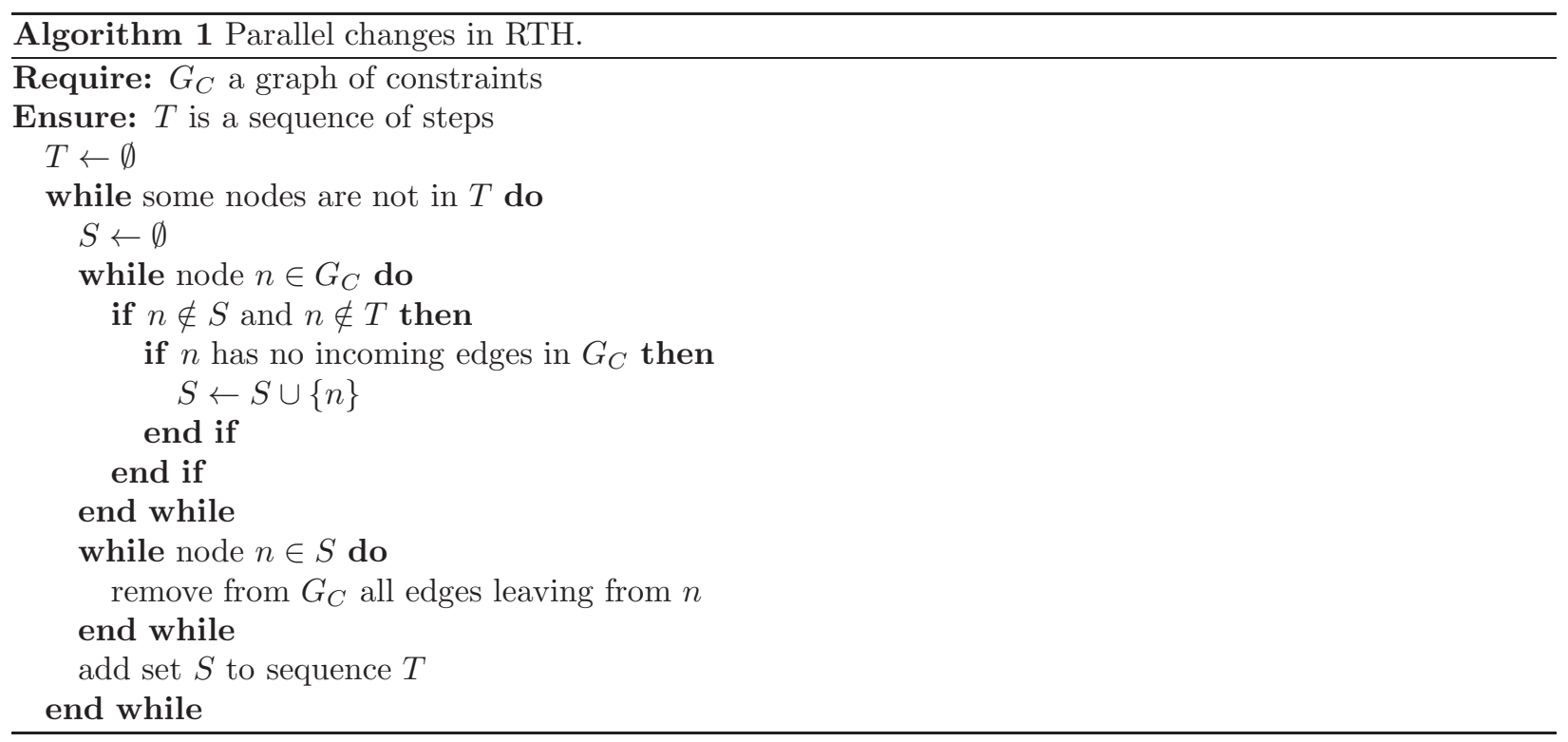

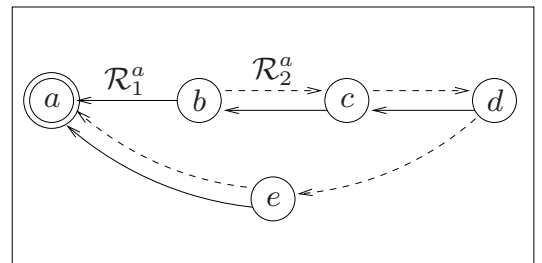

(a)

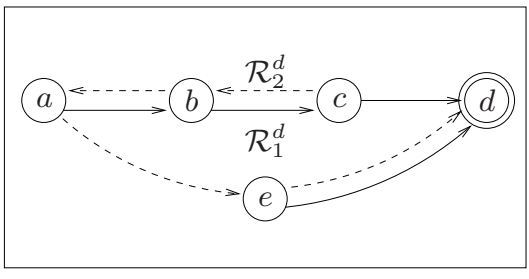

(b)

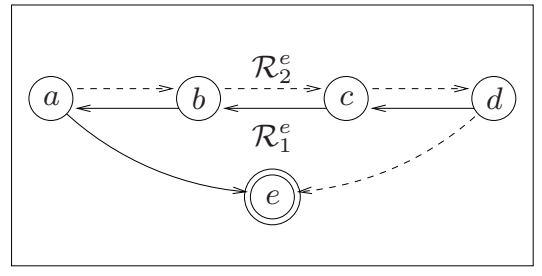

(c)

Figure 2: Example of routing protocols for three destinations: (a) $a$ is the destination, (b) $d$ is the destination, and (c) $e$ is the destination. 


\subsection{Problem formulation for the construction of sequence of steps}

Let $V$ be a set of nodes and $d \in V$ a destination. Let us consider two routing protocols for destination $d$ : $\mathcal{R}_{1}$ is the routing protocol initially used by nodes, and $\mathcal{R}_{2}$ is the new protocol to use. For all $i \in\{1,2\}$ and for all $n \in V$, we denote by $\mathcal{R}_{i}(n) \in V$ the next-hop of $n$ towards $d$ according to $\mathcal{R}_{i}$. Given a partition $\left(V_{1}, V_{2}\right)$ of $V$ (i.e., $V_{1} \cup V_{2}=V$ and $V_{1} \cap V_{2}=\emptyset$ ), we denote by $\mathcal{R}_{V_{1}, V_{2}}$ the routing protocol defined in the following way: for all $n \in V_{1}, \mathcal{R}_{V_{1}, V_{2}}(n)=\mathcal{R}_{1}(n)$, and for all $n \in V_{2}, \mathcal{R}_{V_{1}, V_{2}}(n)=\mathcal{R}_{2}(n)$. In other words, nodes of $V_{1}$ route according to $\mathcal{R}_{1}$, and nodes of $V_{2}$ route according to $\mathcal{R}_{2}$. The set of routing decisions of both $\mathcal{R}_{1}$ and $\mathcal{R}_{2}$ form the set $E$ of the edges of the graph $G=(V, E)$.

Definition 1 (Loop-free step). Let $\left(V_{1}, V_{2}\right)$ be a partition of nodes, and $S \subset V_{1}$ a set of nodes that change their routing protocol to $\mathcal{R}_{2}$ in arbitrary order. $S$ is called step. Step $S$ is said to be loop-free if and only if for all $S^{\prime} \subset S$, the routing protocol $\mathcal{R}_{V_{1} \backslash S^{\prime}, V_{2} \cup S^{\prime}}$ is loop-free.

Definition 1 states that a step $S$ is loop-free if and only if all the possible intermediate sub-steps $S^{\prime} \subset S$ correspond to a loop-free routing protocol. This means that the nodes of $S$ can change their routing protocol arbitrarily without causing loops.

Definition 2 (Loop-free sequence). Let $T=\left(S_{1}, \ldots, S_{m}\right)$ be a sequence of steps. Sequence $T$ is said to be loop-free if and only if both conditions apply:

- $\left(S_{1}, S_{2}, \ldots, S_{m}\right)$ partitions the set $V$,

- for all $i \in[1 ; m], S_{i}$ is a loop-free step on partition $\left(V_{1}^{i}, V_{2}^{i}\right)$, with $V_{2}^{i}=S_{1} \cup \ldots \cup S_{i-1}$ and $V_{1}^{i}=V \backslash V_{2}^{i}$.

Definition 2 states that a sequence $T=\left(S_{1}, \ldots, S_{m}\right)$ is loop-free if and only if each step $S_{i}$ is loop-free, and after step $S_{m}$, all nodes belong to $V_{2}^{m} \cup S_{m}=V$ (that is, they have changed to the target routing protocol $\mathcal{R}_{2}$ ). Note that the set of nodes running $\mathcal{R}_{2}$, which is $V_{2}^{i}$, increases as the sequence progresses.

Figure 3 shows an example of loop-free sequence $T=(\{a, b, d\},\{c\})$. Figure 3(a) shows the initial routing protocol $\mathcal{R}_{1}$. Figure $3(\mathrm{~b})$ shows both $\mathcal{R}_{1}$ and $\mathcal{R}_{2}$ for nodes of the first step $\{a, b, d\}$, to indicate that these nodes might route either according to $\mathcal{R}_{1}$ (if they have not changed yet) or $\mathcal{R}_{2}$ (if they have already changed). It can be seen that for any routing protocol used by the nodes of the first step, no loop occurs for any arbitrary order of change. Figure $3(\mathrm{c})$ shows $\mathcal{R}_{2}$ for nodes that have changed their routing protocol on the previous step, and shows both $\mathcal{R}_{1}$ and $\mathcal{R}_{2}$ for the node of the second step. On a side note, it can be noticed that $T$ is a loop-free sequence with a minimum number of steps, as it is not possible to have both $b$ and $c$ in the same loop-free step.

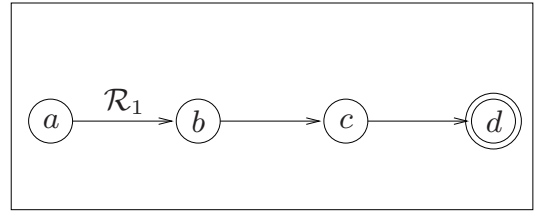

(a)

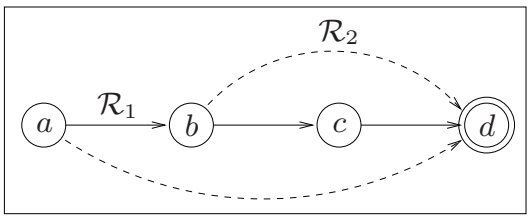

(b)

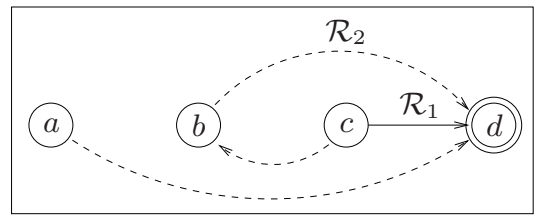

(c)

Figure 3: An example of loop-free sequence $T=(\{a, b, d\},\{c\})$. (a) Initially, all nodes route according to $\mathcal{R}_{1}$. (b) During the first step, nodes from $\{a, b, d\}$ might route according to $\mathcal{R}_{1}$ or $\mathcal{R}_{2}$ without causing loops. (c) During the second step, node $c$ routes according to $\mathcal{R}_{1}$ or $\mathcal{R}_{2}$, while all nodes from $\{a, b, d\}$ route according to $\mathcal{R}_{2}$. At the end of the second step, all nodes route according to $\mathcal{R}_{2}$ (not shown).

Theorem 1. Let $G, V_{1}, V_{2}, \mathcal{R}_{1}$ and $\mathcal{R}_{2}$ be defined as previously for a destination d. Let $G^{\prime}=\left(V, E^{\prime}\right)$ with $E^{\prime} \subset E$, such that for all $x \in V_{1},\left(x, \mathcal{R}_{1}(x)\right) \in E^{\prime}$ and $\left(x, \mathcal{R}_{2}(x)\right) \in E^{\prime}$, and for all $x \in V_{2},\left(x, \mathcal{R}_{2}(x)\right) \in E^{\prime}$. Let $\mathcal{C}=\left\{\mathcal{C}_{i}\right\}_{i}$ be the set of all strongly connected components of $G^{\prime}$. For all $i$, let us denote by $\mathcal{C}_{i}^{\prime} \subset \mathcal{C}_{i}$ a set of nodes that verifies the following properties:

- $\mathcal{C}_{i}^{\prime} \subset V_{1}$, 
- $G_{i}^{\prime}=\left(V, E_{i}^{\prime}\right)$ does not contain any loop, with $E_{i}^{\prime}$ defined as follows:

- if $x \in \mathcal{C}_{i}^{\prime}$ then $\left(x, \mathcal{R}_{1}(x)\right) \in E_{i}^{\prime}$ and $\left(x, \mathcal{R}_{2}(x)\right) \in E_{i}^{\prime}$,

- if $x \in V_{1} \backslash \mathcal{C}_{i}^{\prime}$ then $\left(x, \mathcal{R}_{1}(x)\right) \in E_{i}^{\prime}$,

- if $x \in V_{2}$ then $\left(x, \mathcal{R}_{2}(x)\right) \in E_{i}^{\prime}$.

Then, $S=\cup_{i} \mathcal{C}_{i}^{\prime}$ is a valid step for destination $d$.

Proof. Let us assume that $\left\{\mathcal{C}_{i}^{\prime}\right\}_{i}$ verifies the properties. We have to show that $S=\cup_{i} \mathcal{C}_{i}^{\prime}$ is a valid step, that is, for every $S^{\prime} \subset S, \mathcal{R}_{V_{1} \backslash S^{\prime}, V_{2} \cup S^{\prime}}$ is loop-free and leads to destination $d$. Let $S^{\prime}$ be an arbitrary subset of $S$, and let us build $\mathcal{R}_{V_{1} \backslash S^{\prime}, V_{2} \cup S^{\prime}}$.

Let us first show that $\mathcal{R}_{V_{1} \backslash S^{\prime}, V_{2} \cup S^{\prime}}$ is loop-free. By contradiction, let us suppose that there is a loop $\left(x_{0}, x_{1}, \ldots, x_{n}\right)$ in $\mathcal{R}_{V_{1} \backslash S^{\prime}, V_{2} \cup S^{\prime}}$, with $x_{n}=x_{0}$.

- Suppose here that this loop spans a single strongly connected component $\mathcal{C}_{i}$. For all $k$, we have $\left(x_{k}, x_{k+1}\right) \in E_{S^{\prime}}$. (i) If $x_{k} \in S^{\prime}$, then $x_{k+1}=\mathcal{R}_{2}\left(x_{k}\right)$ by construction of $E_{S^{\prime}}$ (since $S^{\prime} \subset S \subset \mathcal{C}_{i}^{\prime}$ ). Because of the properties of $\mathcal{C}_{i}^{\prime}$, we have $\left(x_{k}, \mathcal{R}_{2}\left(x_{k}\right)\right) \in E_{i}^{\prime}$. (ii) If $x_{k} \notin S^{\prime}$ and $x_{k} \in V_{1}$, then $x_{k+1}=\mathcal{R}_{1}\left(x_{k}\right)$ by construction of $E_{S^{\prime}}$. We have to consider the two following sub-cases: $x_{k} \in \mathcal{C}_{i}^{\prime}$ and $x_{k} \notin \mathcal{C}_{i}^{\prime}$. If $x_{k} \in \mathcal{C}_{i}^{\prime}$, then $\left(x_{k}, \mathcal{R}_{1}\left(x_{k}\right)\right) \in E_{i}^{\prime}$. If $x_{k} \notin \mathcal{C}_{i}^{\prime}$, then $\left(x_{k}, \mathcal{R}_{1}\left(x_{k}\right)\right) \in E_{i}^{\prime}$. (iii) If $x_{k} \notin S^{\prime}$ and $x_{k} \in V_{2}$, then $x_{k+1}=\mathcal{R}_{2}\left(x_{k}\right)$ by construction of $E_{S^{\prime}}$, and $x_{k} \notin \mathcal{C}_{i}^{\prime}$. Thus, $\left(x_{k}, \mathcal{R}_{2}\left(x_{k}\right)\right) \in E_{i}^{\prime}$. To summarize these three cases, all the arcs of the loop on $E_{S^{\prime}}$ are also included in the arcs of $E_{i}^{\prime}$, thus $G_{i}^{\prime}$ contains a loop. However, this is impossible by construction of $\mathcal{C}_{i}^{\prime}$. Thus, by contradiction, there is no loop on $G_{S^{\prime}}$.

- Suppose now that this loop spans several strongly connected components, including $\mathcal{C}_{i}$ and $\mathcal{C}_{j}$, with $i \neq j$. For all nodes $x_{m}$ of the loop (with $\left(x_{m}, x_{m+1}\right) \in E_{S^{\prime}}$ ), let us show that $\left(x_{m}, x_{m+1}\right) \in E^{\prime}$. (i) If $x_{m} \in S^{\prime}$, then $x_{m+1}=\mathcal{R}_{2}\left(x_{m}\right)$, and $x_{m} \in \mathcal{C}_{i}^{\prime}$, which means that $x_{m} \in V_{1}$. Thus, $\left(x_{m}, \mathcal{R}_{2}\left(x_{m}\right)\right) \in E^{\prime}$ by construction of $E^{\prime}$. (ii) If $x_{m} \notin S^{\prime}$ and $x_{m} \in V_{1}$, then $x_{m+1}=\mathcal{R}_{1}\left(x_{m}\right)$. Thus, $\left(x_{m}, x_{m+1}\right) \in E^{\prime}$. (iii) If $x_{m} \notin S^{\prime}$ and $x_{m} \in V_{2}$, then $x_{m+1}=\mathcal{R}_{2}\left(x_{m}\right)$. Thus, $\left(x_{m}, x_{m+1}\right) \in E^{\prime}$. To summarize these three cases, for all $x_{m}$ of the loop, $\left(x_{m}, x_{m+1}\right) \in E^{\prime}$, so there exists a loop in $G^{\prime}$ between a node of $\mathcal{C}_{i}$ and a node of $\mathcal{C}_{j}$, which means that $\mathcal{C}_{i}$ and $\mathcal{C}_{j}$ are the same strongly connected component, which is impossible.

Let us show now that for any node $x, d$ is reachable from $x$ in $G_{S^{\prime}}$. By contradiction, let us suppose that there is a node $x$ from which $d$ is not reachable. Let us consider the path starting from $x$ in $G_{S^{\prime}}$. Either there exists a node on the path that has no next-hop on $G_{S^{\prime}}$, or the path has a loop. We just proved that there is no loop in $G_{S^{\prime}}$, so there has to be a node $y$ without a next-hop. By construction of $G_{S^{\prime}}$, we can see that all nodes $y \in S^{\prime} \cup\left(V_{1} \backslash S^{\prime}\right) \cup V_{2}=V$ have a next-hop, so destination $d$ is reachable from any node $x$ in $G_{S^{\prime}}$. This completes the proof.

Figure 4 shows an example of a graph of nine nodes, where destination is node $f$. The routing protocols $\mathcal{R}_{1}$ and $\mathcal{R}_{2}$ are shown on Figure 4(a). To build the first valid step, all routing arcs are considered. The strongly connected components of the resulting graph are the following: $\mathcal{C}_{1}=\{f\}, \mathcal{C}_{2}=\{b, c\}$ and $\mathcal{C}_{3}=\{a, d, e, g, h, i\}$. The following sets verify the property of the theorem: $\mathcal{C}^{\prime}{ }_{1}=\{f\}, \mathcal{C}^{\prime}{ }_{2}=\{b\}$ and $\mathcal{C}^{\prime}{ }_{3}=\{d, g, h, i\}$. Indeed, it can be verified that none of the graphs $G_{i}^{\prime}$ (for $i \in\{1,2,3\}$ ) has a loop (this can also be seen on the graph shown on Figure 4(b)). Thus, the step $S_{1}=\{b, d, f, g, h, i\}$ is a valid first step. To build the second valid step, the strongly connected components of the graph shown on Figure 4(c) are computed. They are the following: $\mathcal{C}_{1}=\{a\}, \mathcal{C}_{2}=\{b\}, \ldots, \mathcal{C}_{9}=\{i\}$. The following sets verify the property of the theorem: $\mathcal{C}_{1}^{\prime}=\{a\}, \mathcal{C}_{3}^{\prime}=\{c\}, \mathcal{C}_{5}^{\prime}=\{e\}$, and $\mathcal{C}_{i}^{\prime}=\emptyset$ otherwise. The second step is $S_{2}=\{a, c, e\}$. After this step, all nodes route according to $\mathcal{R}_{2}$. Thus, $T=\left(S_{1}, S_{2}\right)$ is a loop-free sequence.

\subsection{Centralized heuristic for loop-free change of routing protocols}

In this subsection, we describe our centralized heuristic for loop-free change, called Strongly Connected component Heuristic with parallel changes ( $\mathrm{SCH}-\mathrm{p})$. We assume that a centralized entity knows the whole network topology, as well as $\mathcal{R}_{1}$ and $\mathcal{R}_{2}$ for each destination. 


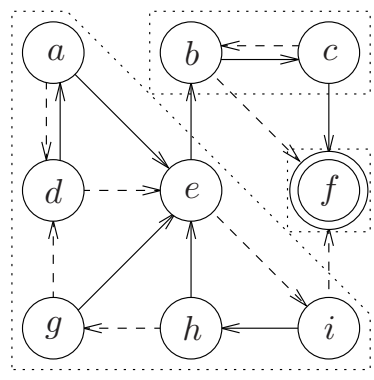

(a)

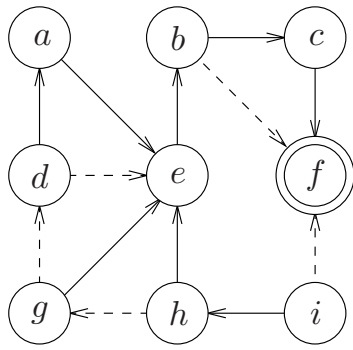

(b)

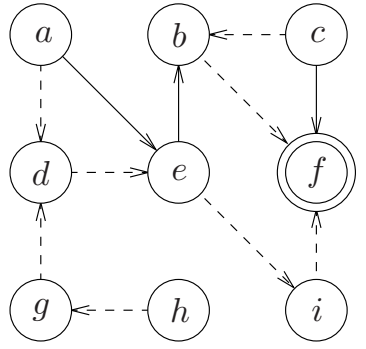

(c)

Figure 4: Sequence based on strongly connected components. (a) The graph has three strongly connected components $\mathcal{C}_{1}=\{f\}$, $\mathcal{C}_{2}=\{b, c\}, \mathcal{C}_{3}=\{a, d, e, g, h, i\}$. (b) The first step is $S_{1}=\{b, d, f, g, h, i\}$, and only the nodes of $S_{1}$ can route according to $\mathcal{R}_{1}$ or $\mathcal{R}_{2}$. (c) The second step is $S_{2}=\{a, c, e\}$, and only the nodes of $S_{2}$ can route according to $\mathcal{R}_{1}$ or $\mathcal{R}_{2}$.

SCH-p is based on Algorithm 2. Each destination is considered sequentially and independently. First, the set of strongly connected components of the graph is built based on the two routing protocols for the current destination. Then, each strongly connected component $\mathcal{C}_{i}$ is considered individually, and nodes are distributed into several steps according to Algorithm 3. We use a greedy approach: for each step $S_{j}$, we add nodes one by one into a set $\mathcal{C}_{i, j}^{\prime}$, until it is not possible to add more nodes without violating the constraints of Theorem 1. Then, we move to the next step, until all nodes of $\mathcal{C}_{i}$ are in a step for this destination.

The worst-case complexity of SCH-p is $\mathcal{O}\left(|V|^{5}\right)$. Indeed, there are at most $|V|$ destinations. For each destination, the strongly connected components of a graph can be computed in $\mathcal{O}(|V|+|E|)$ with Tarjan's algorithm [19]. Note that in our graphs, $|E| \leq 2|V|$ as each node has at most two outgoing arcs (one with $\mathcal{R}_{1}$ and one with $\mathcal{R}_{2}$ ). For each strongly connected component $\mathcal{C}_{i}$, there are at most $\left|\mathcal{C}_{i}\right|$ resulting steps (as there is at least one node per step). Computing the set $\mathcal{C}_{i, j}^{\prime}$ requires considering at most $\left|\mathcal{C}_{i}\right|^{2}$ combinations of nodes, each requiring to build $G^{\prime}$ and determining if it has a loop, which can be done in $\mathcal{O}\left(\left|\mathcal{C}_{i}\right|\right)$. As the set of strongly connected components partitions the graph, we obtain the overall complexity of $\mathcal{O}\left(|V|^{5}\right)$.

\section{Simulation results}

In this section, we describe our simulation results. We start by describing our settings. Then, we quantify the probability of loop occurrence when using arbitrary routing protocols, with uncontrolled changes (that is, without any heuristic to avoid transient loops). We also quantify the number of troublesome destinations for RTH and RTH-p. Then, we compute the number of steps required to change the routing protocols for RTH, RTH-p and our heuristic SCH-p.

\subsection{Topologies and parameter settings}

We used two types of topologies in order to evaluate the heuristics over a large number of networks. First, we decided to generate random connected graphs. We generated networks composed of 50, 100, 150, 200, 250 , and 300 nodes, randomly deployed on an area of $100 \mathrm{~m} \times 100 \mathrm{~m}$. Nodes that are distant of less than $20 \mathrm{~m}$ are considered connected. Second, we decided to test our heuristic SCH-p on real networks. We used the Rocketfuel network topologies [20,21], which are also used for the evaluation of RTH in [8]. The resulting topologies have $79,87,104,138,161$, and 315 nodes.

In the following, we use three scenarios for the routing protocols. They are all based on shortest paths, using different link metrics. In Scenario $1, \mathcal{R}_{1}$ uses the hop-count metric and $\mathcal{R}_{2}$ uses a random metric, chosen randomly within $[1 ; 100]$ for each link. $\mathcal{R}_{2}$ models a protocol based on a delay or loss rate. In Scenario 2, both $\mathcal{R}_{1}$ and $\mathcal{R}_{2}$ are based on independent random metrics chosen within $[1 ; 100]$ (such as delay for $\mathcal{R}_{1}$ and loss rate for $\mathcal{R}_{2}$ ). In Scenario $3, \mathcal{R}_{1}$ is based on a random metric chosen within $[1 ; 100]$ for each link, and $\mathcal{R}_{2}$ uses a correlated weight for links. If $w \in[1 ; 100]$ denotes the weight of the link for $\mathcal{R}_{1}$, the weight of the link for $\mathcal{R}_{2}$ is chosen randomly within $[\max (1, w-10) ; \min (100, w+10)]$. This models the 

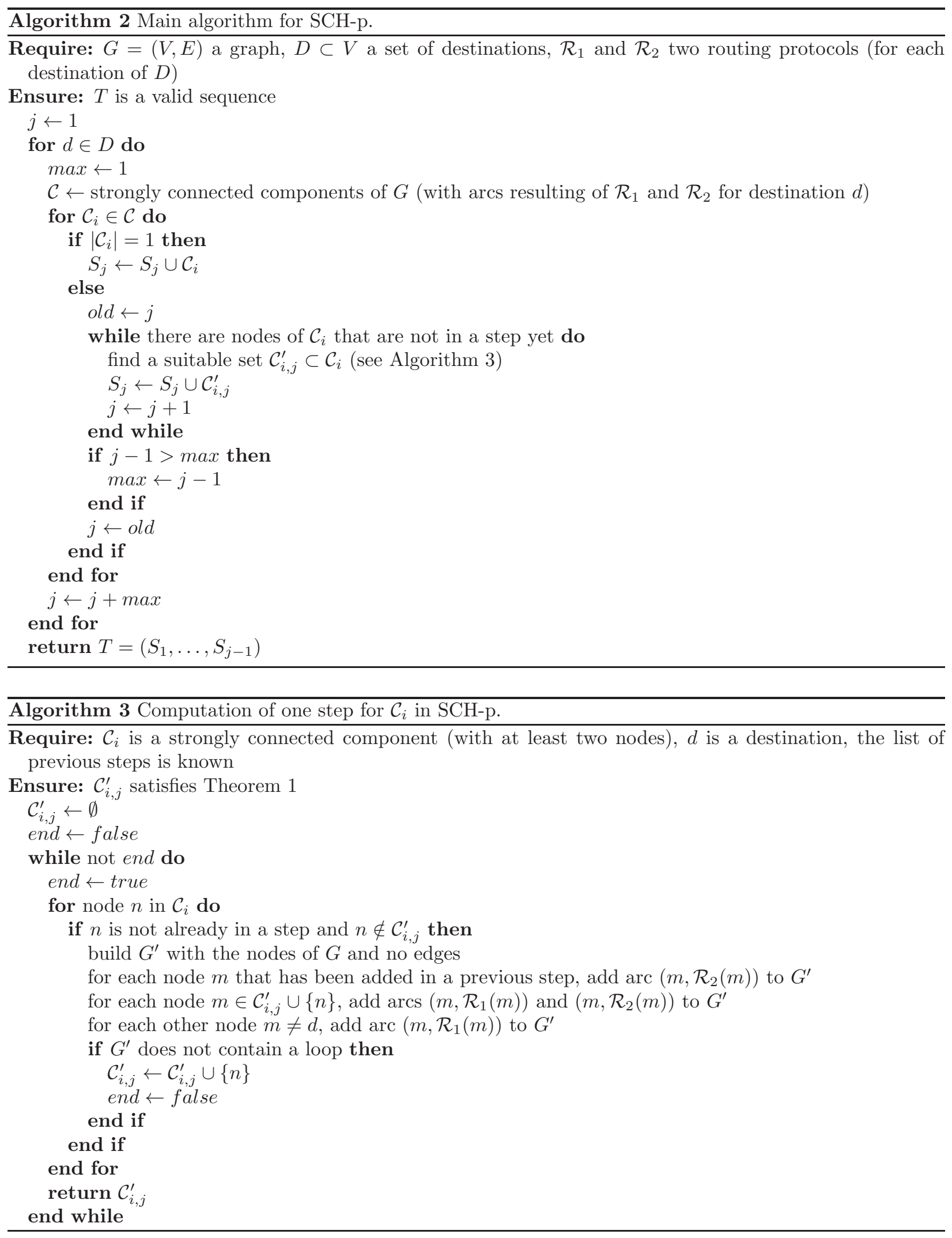
case where protocol $\mathcal{R}_{2}$ uses an updated version of the topology, or includes an additional parameter in the computation of link weights.

\subsection{Simulation on loop occurrence with uncontrolled changes}

We consider that a loop occurs if it is possible for a packet to enter a routing loop when nodes on the path decide arbitrarily to route according to $\mathcal{R}_{1}$ or $\mathcal{R}_{2}$. For instance, we consider that there is a loop in the topology shown on Figure 1 , because it is possible that $b$ routes according to $\mathcal{R}_{1}$ while $c$ routes according to $\mathcal{R}_{2}$. Notice that even if there is a loop occurrence in a topology, some nodes might be able to send packets to the destination without loops. Thus, our metric refers to the risk of occurrence of at least one loop.

In the following, all the results on loop occurrences are averaged over 100 simulations per destination, and over all possible destinations.

\subsubsection{Loop occurrences on random networks}

In this subsubsection, we quantify the probability of loop occurrence for random graphs, in the three previous scenarios.

Figure 5 shows the percentage of loop occurrence as a function of the number of nodes in the network, for the three scenarios. In Scenario 1, the percentage of loop occurrence increases with the number of nodes. Even when the number of nodes is small (for instance, 50), the percentage of loop occurrence is about $60 \%$, which means that loops are likely to occur. This comes from the fact that the two routing protocols build paths with low correlations due to the random weight of $\mathcal{R}_{2}$. In Scenario 2, the percentage of loop occurrence is about $100 \%$ for all numbers of nodes in the network. This is due to the fact that $\mathcal{R}_{1}$ and $\mathcal{R}_{2}$ yield to different routing decisions, thus paths have a very low correlation. In Scenario 3, the percentage of loops is low for a small number of nodes. Indeed, since the link metrics are correlated, $\mathcal{R}_{1}$ and $\mathcal{R}_{2}$ are likely to be similar, so packets are likely to follow similar paths. However, when the number of nodes is large, the two routing protocols exhibit differences, and the resulting shortest paths have low correlations (even if the weights are correlated).

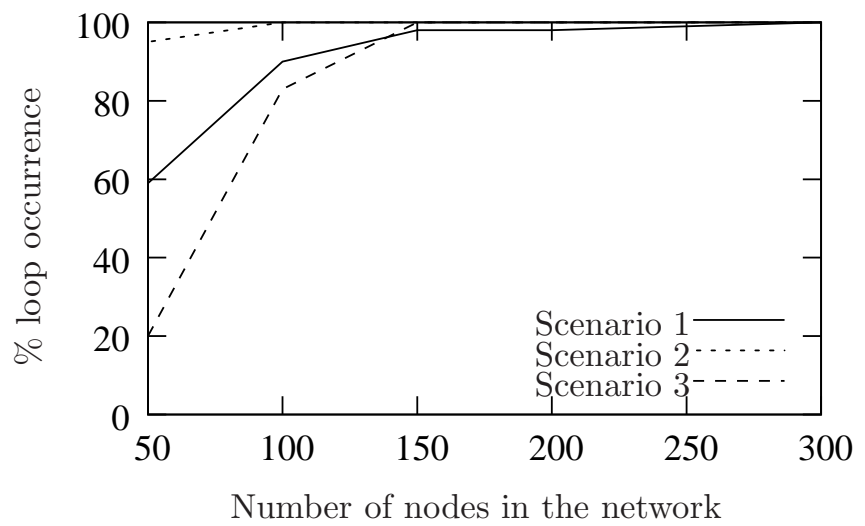

Figure 5: In random networks, when $\mathcal{R}_{1}$ is based on a hop-count metric and $\mathcal{R}_{2}$ is based on a random metric, the percentage of loop occurrence is high, due to the low correlation of the metrics of $\mathcal{R}_{1}$ and $\mathcal{R}_{2}$. When $\mathcal{R}_{1}$ and $\mathcal{R}_{2}$ are both based on a random metric, the percentage of loop occurrence is very high, again due to the very low correlation of the metrics of $\mathcal{R}_{1}$ and $\mathcal{R}_{2}$. When $\mathcal{R}_{1}$ is based on a random metric and $\mathcal{R}_{2}$ is based on a deviation of the metric of $\mathcal{R}_{1}$, the percentage of loop occurrence is high for topologies with a large number of nodes, despite the high correlation of the metrics.

\subsubsection{Loop occurrences on real networks}

In this subsubsection, we quantify the number of loops that might appear using $\mathcal{R}_{1}$ and $\mathcal{R}_{2}$, for real networks.

Figure 6 shows the percentage of loop occurrence as a function of the number of nodes, for the three scenarios. We notice that the percentage of loop occurrence is much larger with Scenario 1 and Scenario 2 
than with Scenario 3. This is due to the fact that the routing protocols $\mathcal{R}_{1}$ and $\mathcal{R}_{2}$ of Scenario 3 are highly correlated. However, the percentage of loop occurrence is more important for large networks as the paths are longer. For real networks, the percentage of loop occurrence depends on the underlying topology. In general, this percentage increases with the network size, but there are some real topologies (such as the Rocketfuel topology '1221.city' with 104 nodes) that yield few routing loops (see also Figure 16 of [8]).

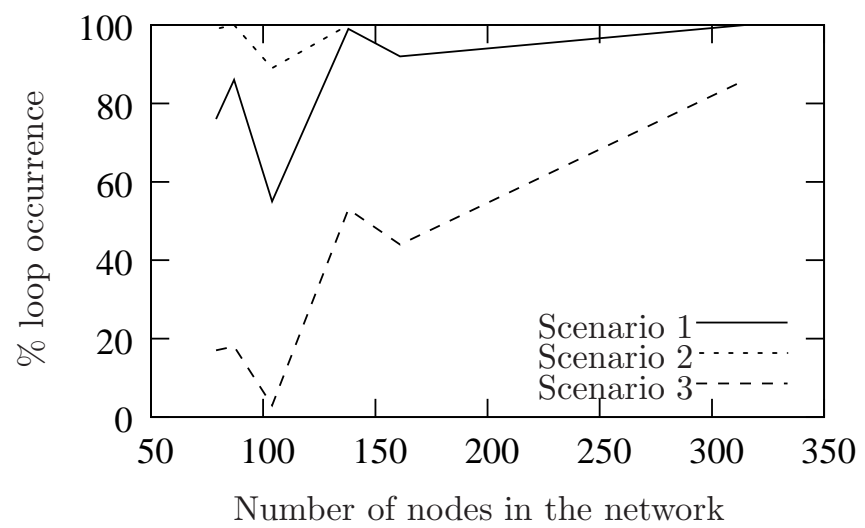

Figure 6: In real networks, for Scenario 1, the percentage of loops is high (between $50 \%$ and $80 \%$ for small networks (less than 104 nodes), and above $90 \%$ for large networks). For Scenario 2, the percentage of loops is always high independently of the network size (between $90 \%$ and 100\%). For Scenario 3, the percentage of loops increases with the network size (10\% for small networks and up to $90 \%$ for large networks).

\subsection{Average percentage of troublesome destinations}

In this subsection, we focus on the percentage of troublesome destinations. Troublesome destinations are destinations that require to be considered independently by RTH and RTH-p. These destinations cannot follow the common per-router ordering used for all the remaining destinations.

We compute the number of troublesome destinations for random and real networks by averaging over 100 simulations, and we normalize it with respect to the number of nodes of the network in order to obtain a percentage. All nodes act as destinations.

\subsubsection{Troublesome destinations for random networks}

Figure 7 shows the average percentage of troublesome destinations in random networks. We notice that the percentage of troublesome destinations is low for small networks and increases consistently for large networks. This is due to the fact that the percentage of loop occurrence is small (cf Subsubsection 4.2.1). Moreover, the percentage of troublesome destinations depends on the scenarios. In Scenario 1, we notice that the percentage of troublesome destinations is low for small networks (less than 26\%) but it increases quickly for large networks (up to 94\%). In Scenario 2, the percentage of troublesome destinations is about $80 \%$ for a network of 50 nodes and goes up to about $100 \%$ for a network of 300 nodes. In Scenario 3, the percentage of troublesome destinations is almost zero for small networks when $\mathcal{R}_{1}$ and $\mathcal{R}_{2}$ are highly correlated, but it increases up to $98 \%$ for large networks.

\subsubsection{Troublesome destinations for real networks}

Figure 8 shows the percentage of troublesome destinations in real networks. For Scenario 1 and Scenario 2, the percentage of troublesome destinations is important (between $70 \%$ and $100 \%$ for Scenario 1, and above $90 \%$ for Scenario 2) for large networks. This percentage is low for small networks as paths to destinations are smaller, which leads to a small number of loops in the network (cf Subsubsection 4.2.2). For Scenario 3, the percentage of troublesome destinations is low, even for large networks. Note that the low percentage of troublesome destinations for Scenario 3 is the main argument used by authors of [8] to justify that there are few troublesome destinations. However, we believe that Scenario 3 represents only a specific case, where the two routing protocols are highly correlated. 


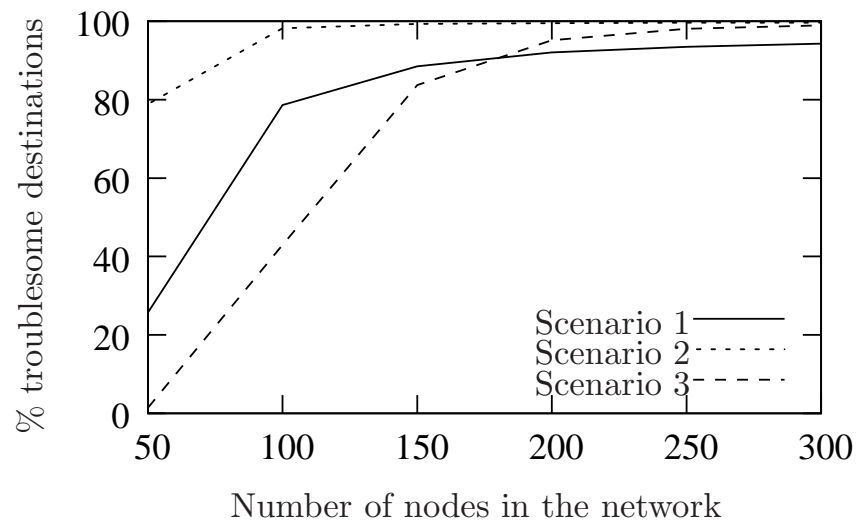

Figure 7: In random networks, the percentage of troublesome destinations is more important in large networks than in small networks. Troublesome destinations appear even with correlated routing protocols (such as in Scenario 3).

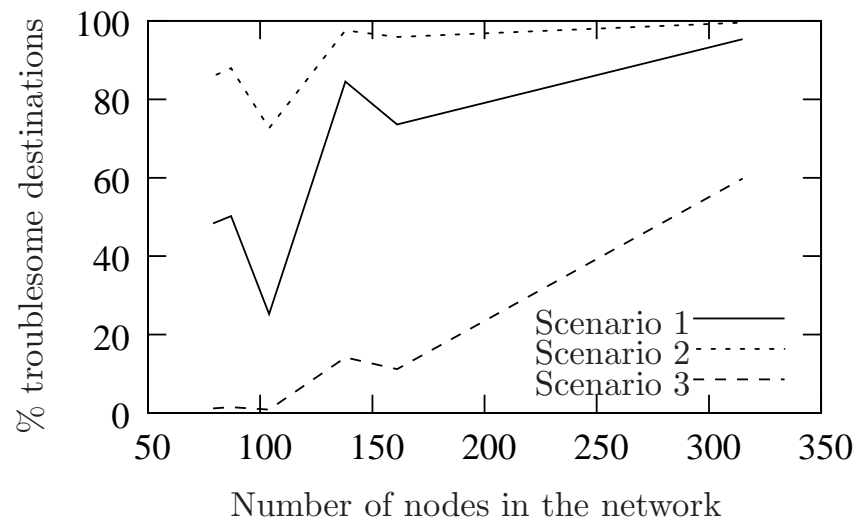

Figure 8: In real networks, the percentage of troublesome destinations is more important in large networks than in small networks. Correlated routing protocols (see Scenario 3) are able to greatly reduce the number of troublesome destinations. 


\subsection{Results on average number of steps}

In this subsection, we evaluate the number of steps required for all the nodes to change from $\mathcal{R}_{1}$ to $\mathcal{R}_{2}$ without any loop occurrence. This is our main metric, as the change duration is proportional to the number of steps produced by the heuristics.

We consider that all nodes are destinations. Simulation results are averaged over 100 repetitions and confidence intervals of $95 \%$ are shown.

We compare our heuristic SCH-p to RTH [8]. Recall that RTH provides an ordering to change the nodes sequentially: the number of steps of RTH is equal to the number of nodes in the network, if there is no troublesome destination. We also compare our heuristic SCH-p to RTH-p. Recall that RTH-p consists of changing several nodes in parallel.

\subsubsection{Number of steps for random networks}

Figure 9 shows the average number of steps required to change the routing protocol, in term of the number of nodes in the network, for Scenario 1. The y-axis is depicted using a logarithmic scale. We notice that the number of step increases consistently with the size of the network for all the heuristics (RTH, RTH-p and SCH-p). We notice also that the number of steps is very large for RTH. This is due to the fact that only one node at each step is able to change from $\mathcal{R}_{1}$ to $\mathcal{R}_{2}$. More precisely, the number of steps for RTH is equal to the number of nodes in the network, times $\alpha+1$, where $\alpha$ is the number of troublesome destinations. Both RTH-p and SCH-p outperform RTH with a gain that reaches up to $97 \%$ for RTH-p, and up to $99 \%$ for SCH-p. SCH-p outperforms RTH-p: for large networks (more than 100 nodes), SCH-p shows a gain of $63 \%$ for a network of 100 nodes, and a gain of $77 \%$ for a network of 300 nodes. The large gain of SCH-p can be explained as follows:

- SCH-p is able to change the routing protocol of nodes independently on their position on the paths from the sources to the destinations (according to $\mathcal{R}_{2}$ ), while RTH-p only changes the routing protocol for nodes starting from the destinations and going backward to the sources.

- Since the number of troublesome destinations is large (see Fig. 7), RTH-p is not able to aggregate many non-troublesome destinations together.

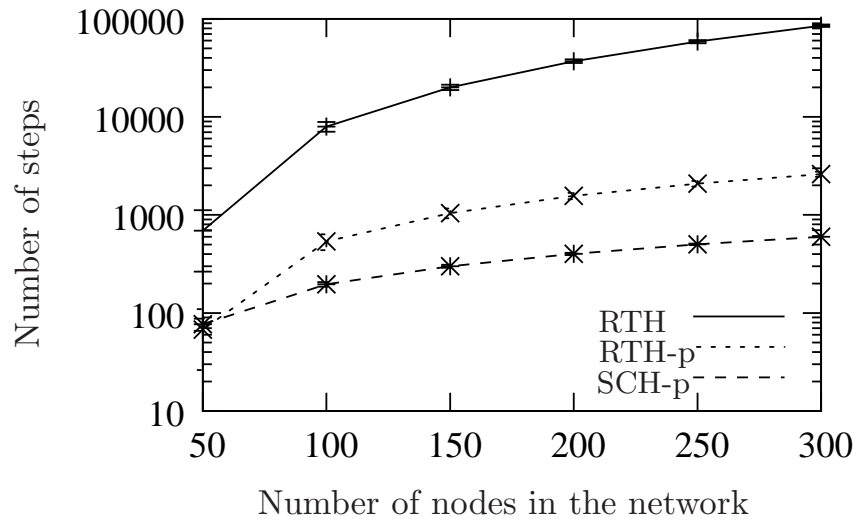

Figure 9: In random networks for Scenario 1, SCH-p is able to perform the change in about 600 steps on average for large networks. RTH and RTH-p require respectively 85182 steps and 2602 steps to perform the change of all the nodes.

Figure 10 shows the average number of steps required to change the routing protocols in term of the number of nodes, for Scenario 2. The y-axis is depicted using a logarithmic scale. We can see that the number of steps increases consistently with the size of the network. We notice also the same behavior for RTH as in Fig. 9: the number of steps required to change all the nodes is very large, especially for large networks. RTH produces 2021 steps for networks of 50 nodes, and 90000 steps for networks of 300 nodes. 


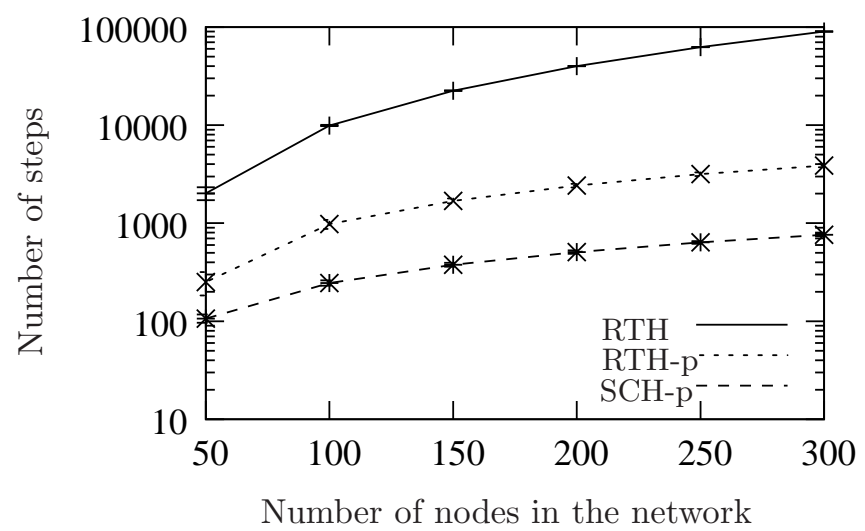

Figure 10: In random networks and for Scenario 2, SCH-p is able to perform the change in about 761 steps on average for large networks. RTH and RTH-p require respectively 90000 steps and 3864 steps to perform the change of all the nodes.

SCH-p outperforms RTH-p (respectively RTH), with a gain varying from 57\% (respectively 94\%) for small networks up to $80 \%$ (respectively $99 \%$ ) for large networks.

Figure 11 shows the average number of steps in term of the number of nodes in the network, for Scenario 3. The y-axis is depicted using a logarithmic scale. RTH-p shows a better behavior than SCH-p for small networks of less than 150 nodes. Indeed, RTH-p shows a gain of $89 \%$ for a network of 50 nodes and $11 \%$ for a network of 100 nodes compared to SCH-p. Those gains are due to the fact that there are few troublesome destinations in this case, as $\mathcal{R}_{1}$ and $\mathcal{R}_{2}$ are highly correlated. Recall that SCH-p considers destinations independently, and thus is not able to benefit from non-troublesome destinations. SCH-p is better than RTH-p for large networks. Indeed, SCH-p shows a gain of $42 \%$ for a network of 150 nodes and of $61 \%$ for a network of 300 nodes, compared to RTH-p.

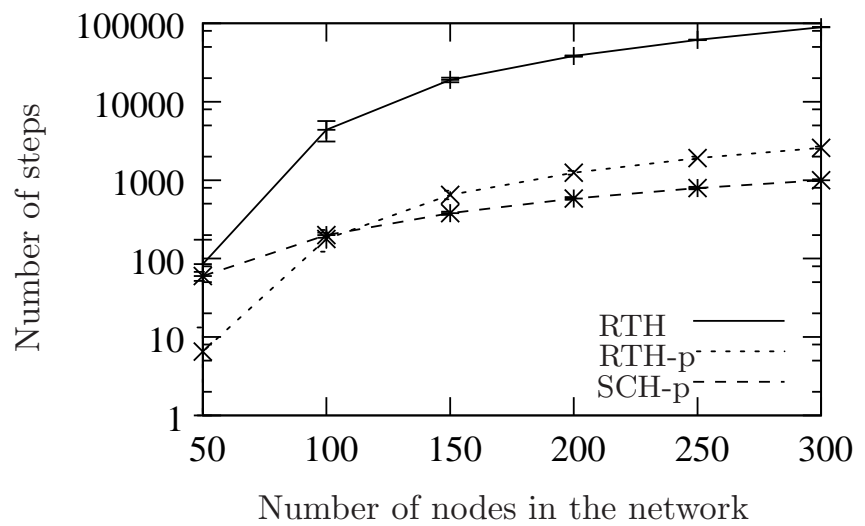

Figure 11: In random networks and for Scenario 3, SCH-p is able to perform the change in about 998 steps on average for large networks. RTH and RTH-p require respectively 89361 steps and 2585 steps to perform the change of all the nodes.

\subsubsection{Number of steps for real networks}

Figure 12 shows the average number of steps in term of the number of nodes for Scenario 1 and Rocketfuel topologies. The y-axis is depicted using a logarithmic scale. We notice the same behavior as for random networks. For small networks, SCH-p and RTH-p achieve a similar performance. For large networks, SCH-p shows better performance than RTH-p: SCH-p shows a gain that reaches $99 \%$ compared to RTH and $68 \%$ compared to RTH-p, for a network of 315 nodes.

Figure 13 shows the average number of steps in term of the number of nodes in the network, for Scenario 2, using a logarithmic scale. The gain of SCH-p reaches $96 \%$ for the smallest network compared to RTH, and 


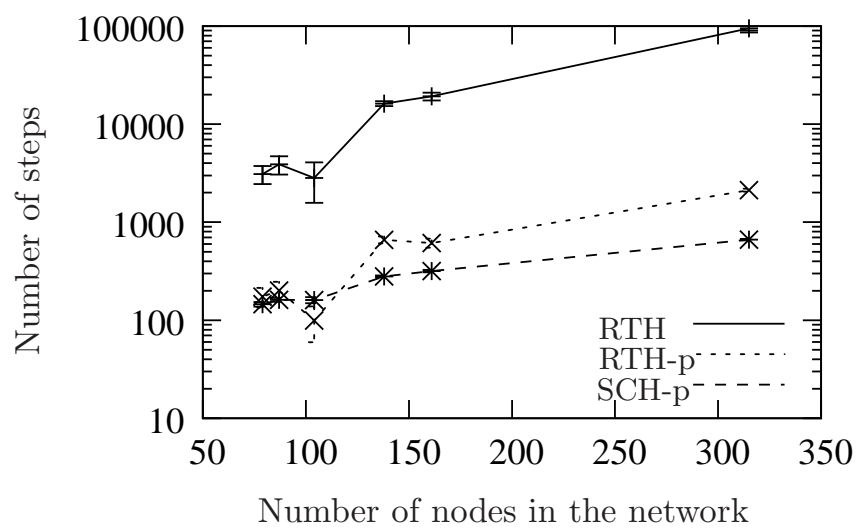

Figure 12: In real networks and for Scenario 1, SCH-p is able to perform the change in about 664 steps on average for the largest network (315 nodes). RTH and RTH-p require respectively 94890 steps and 2121 steps to perform the change of all the nodes.

$48 \%$ compared to RTH-p. The largest gain reached for the largest network is $99 \%$ compared to RTH, and $69 \%$ compared to RTH-p.

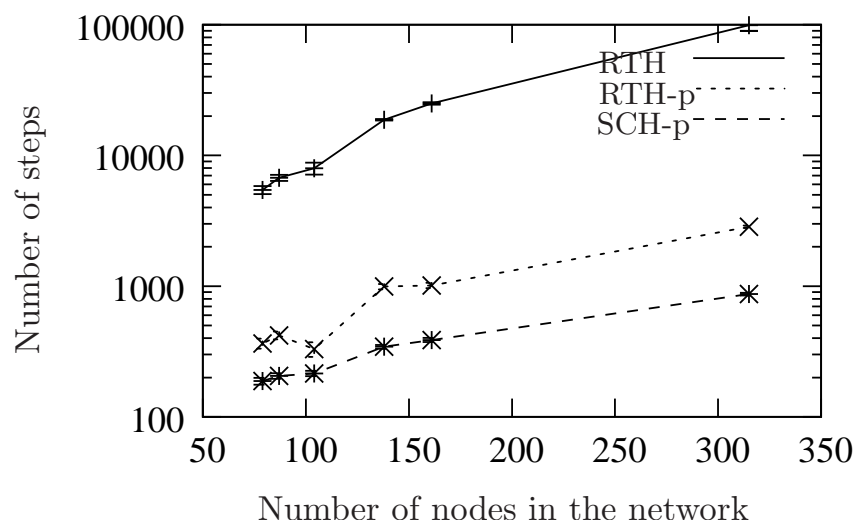

Figure 13: In real networks and for Scenario 2, SCH-p is able to perform the change in about 869 steps on average for the largest network. RTH and RTH-p require respectively 99102 steps and 2851 steps to perform the change of all the nodes.

Figure 14 shows the average number of steps in term of the number of nodes in the network, for Scenario 3 , using a logarithmic scale. RTH-p is better than SCH-p due to the very small number of troublesome destinations in this specific scenario and for these specific networks. The gain of RTH-p reaches $94 \%$ for a network of 104 nodes.

The computation time of SCH-p is larger than the computation time of RTH and RTH-p. With our mono-thread implementation on a i7-2600 CPU at $3.40 \mathrm{GHz}$ (with eight cores), SCH-p is able to compute all the steps for the largest real topology in 91 seconds on average (with a standard deviation of 3 seconds, and for Scenario 2 which yields the largest number of loops). RTH and RTH-p compute all the steps in 1.5 seconds and 2.2 seconds respectively. However, we believe that the computation time of SCH-p is reasonable for large networks of 300 nodes, and that the gain of the change duration outweights the computation time.

\section{Conclusion}

When a routing protocol has to be changed, transient routing loops might occur in the network. In this paper, we quantified the percentage of loop occurrence on several scenarios. We show that loops can be completely avoided by having nodes change their routing protocol according to a sequence which depends 


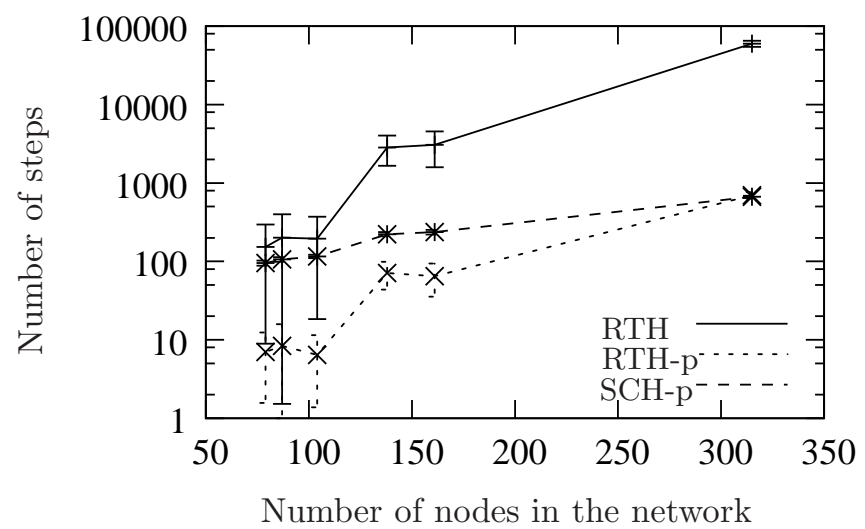

Figure 14: In real networks and for Scenario 3, RTH-p is generally better than SCH-p due to the very low number of troublesome destinations. SCH-p performs the change in about 666 steps for the largest network. RTH and RTH-p require respectively 59629 steps and 698 steps to perform the change of all the nodes.

on the topology and on the routing protocols. This sequence is a list of steps, where each step is a set of nodes that can change their routing protocol in arbitrary order. We proposed RTH-p, an enhancement of the RTH heuristic [8], that is able to change several nodes in parallel. Then, we proposed SCH-p, a centralized heuristic which builds sequences with a small number of steps. Reducing the number of steps is crucial, as the duration of a change is proportional to the number of steps. Simulation results for random and real networks show that SCH-p outperforms both RTH and RTH-p in most scenarios. The gain of SCH-p in term of number of steps varies between $80 \%$ and $99 \%$ when compared to RTH, and between $61 \%$ and $77 \%$ for most large networks when compared to RTH-p.

\section{References}

[1] K. Butler, T. R. Farley, P. McDaniel, J. Rexford, A survey of BGP security issues and solutions, Proceedings of the IEEE 98 (1) (2010) 100-122.

[2] P. Trakadas, T. Zahariadis, S. Voliotis, P. Karkazis, T. Velivassaki, L. Sarakis, Routing metric selection and design for multi-purpose WSN, in: IWSSIP (International Conference on Systems, Signals and Image Processing), 2014, pp. 199-202.

[3] Z. Zhong, R. Keralapura, S. Nelakuditi, Y. Yu, J. Wang, C. N. Chuah, S. Lee, Avoiding transient loops through interface-specific forwarding, in: IEEE/ACM IWQoS (International Symposium on Quality of Service), Vol. 3552 of Lecture Notes in Computer Science, Springer, 2005, pp. 219-232.

[4] P. Francois, O. Bonaventure, Avoiding transient loops during the convergence of link-state routing protocols, IEEE/ACM Transactions on Networking 15 (6) (2007) 1280-1292. doi:10.1109/TNET.2007.902686.

[5] S. Nelakuditi, Z. Zhong, J. Wang, R. Keralapura, C. N. Chuah, Mitigating transient loops through interface-specific forwarding, Computer Networks 52 (3) (2008) 593-609.

[6] N. El Rachkidy, A. Guitton, M. Misson, Avoiding routing loops in a multi-stack WSN, JCM (Journal of Communications) 8 (3) (2013) 751-761.

[7] N. El Rachkidy, A. Guitton, M. Misson, Improving routing performance when several routing protocols are used sequentially in a WSN, in: ICC (IEEE International Conference on Communications), 2013, pp. 1408-1413.

[8] L. Vanbever, S. Vissichio, C. Pelsser, P. Francois, O. Bonaventure, Lossless migrations of link-state IGPs, IEEE/ACM Transactions on Networking 20 (6) (2012) 1842-1855. 
[9] R. Shirani, M. St-Hilaire, T. Kunz, Y. Zhou, J. Li, L. Lamont, Combined reactive-geographic routing for unmanned aeronautical adhoc networks, in: IWCMC (International Wireless Communications and Mobile Computing Conference), 2012.

[10] S. Moad, M. T. Hansen, R. Jurdak, B. Kusy, N. Bouabdallah, A. Ksentini, On balancing between minimum energy and minimum delay with radio diversity for wireless sensor networks, in: IFIP Wireless Days, 2012.

[11] M. Fonoage, M. Cardei, A. Ambrose, A QoS based routing protocol for wireless sensor networks, in: IPCCC (IEEE Performance Computing and Communications Conference), 2010.

[12] J. Steffan, L. Fiege, M. Cilia, A. P. Buchmann, Towards multi-purpose wireless sensor networks, in: Systems Communications, 2005, pp. 336-341.

[13] J. Steffan, M. Voss, Security mechanisms for multi-purpose sensor networks, in: GI Jahrestagung, Vol. 2, 2005, pp. 158-160.

[14] D. Manjunath, S. V. Gopaliah, A multi-purpose wireless sensor network for residential layouts, in: BWCCA (International Conference on Broadband and Wireless Computing, Communication and Applications), 2007, pp. 49-58.

[15] P. Javier del Cid, Optimizing resource use in multi-purpose WSNs, in: PerCom Workshops (Pervasive Computing Workshops), 2011, pp. 395-396.

[16] L. Sarakis, T. Zahariadis, H.-C. Leligou, M. Dohler, A framework for service provisioning in virtual sensor networks, EURASIP Journal on Wireless Communications and Networking (1) (2012) 135.

[17] F. Clad, P. Merindol, J.-J. Pansiot, P. Francois, O. Bonaventure, Graceful convergence in link-state IP networks: A lightweight algorithm ensuring minimal operational impact, IEEE/ACM Transactions on Networking 22 (1) (2013) 300-312. doi:10.1109/TNET.2013.2255891.

[18] F. Clad, P. Merindol, S. Vissicchio, J.-J. Pansiot, P. François, Graceful router updates for link-state protocols, in: IEEE ICNP (International Conference on Network Protocols), 2013.

[19] R. E. Tarjan, Depth-first search and linear graph algorithms, SIAM Journal on Computing 1 (2) (1972) $146-160$.

[20] N. Spring, R. Mahajan, D. Wetherall, Measuring ISP topologies with rocketfuel, in: ACM SIGCOMM, 2002, pp. 133-145.

[21] Rocketfuel project, http://research.cs.washington.edu/networking/rocketfuel/ (2002). 